\title{
A novel fibrinogen mutation: FGA g. 3057 C > T (p. Arg104 > Cys) impairs fibrinogen secretion
}

\author{
R. Marchi ${ }^{1 *}$ (D, M. Linares ${ }^{1}$, H. Rojas ${ }^{2}$, A. Ruiz-Sáez ${ }^{3}$, M. Meyer ${ }^{4}$, A. Casini ${ }^{5}$ and S.O. Brennan ${ }^{6}$
}

\begin{abstract}
Background: Abnormal fibrinogens can be caused by clinically silent hereditary mutations. A new case was detected accidentally in an 11-year-old girl when routine pre-operative coagulation tests were performed for nasal turbinate surgery.
\end{abstract}

Methods: The fibrinogen genes FGA, FGG and FGB were sequenced using standard protocols. The kinetics of fibrin formation were followed by turbidity at $350 \mathrm{~nm}$. Purified fibrinogen was incubated with plasmin, and the degradation products analyzed by SDS/PAGE. The formation of fibrinogen-albumin complexes was analyzed by immunobloting. Fibrin structure was examined in a Nikon Eclipse TE 2000-U laser microscope. Secretion of the variant protein was analyzed directly by reverse phase-electrospray time of flight-mass spectrometry (TOF-MS).

Results: DNA sequencing revealed a novel heterozygous g. $3057 \mathrm{C}>$ T mutation in the FGA that predicts a p. Arg104 > Cys substitution, in the proband and her father. Both patients were asymptomatic with low functional and antigen fibrinogen concentrations. The proband's plasma fibrinogen polymerization was almost normal, with a 12\% decrease in the final turbidity, while, the father's fibrin formation had a diminished slope and final turbidity (2.5x and 40\%, respectively). Aa Arg104 is located at a plasmin cleavage site in the coiled-coil region of fibrinogen. However, the father's fibrinogen plasmin degradation was normal. Although the exchanged Cys introduces an unpaired $-\mathrm{SH}$, immunoblotting showed no fibrinogen-albumin complexes. Furthermore, the plasma clot structure observed by confocal microscopy appeared almost normal. TOF-MS showed that the variant Aa chain was underrepresented in plasma and made up only about $25 \%$ of the total.

Conclusions: The low expression of the Aa Arg104 > Cys chain in circulation could account for the observed hypodysfibrinogenemia.

Keywords: Abnormal fibrinogen, Hypodysfibrinogenemia, Fibrinogen Aa chain

\section{Background}

Fibrinogen is the central protein of blood coagulation. Once the coagulation cascade is initiated, thrombin is formed and catalyzes the conversion of fibrinogen into soluble fibrin monomers that polymerize spontaneously, forming a three-dimensional network that becomes further stabilized by activated factor XIII (FXIIIa).

\footnotetext{
* Correspondence: rmarchi@ivic.gob.ve

${ }^{1}$ Lab. Biología del Desarrollo de la Hemostasia. Instituto Venezolano de Investigaciones Científicas (IVIC), Caracas, Bolivarian Republic of Venezuela Full list of author information is available at the end of the article
}

Polymerization is initiated by cleavage of the A and $\mathrm{B}$ peptides from the $\mathrm{N}$-terminal of the $\mathrm{A} \alpha$ and $\mathrm{B} \beta$ chains [1]. Fibrinogen is a $340 \mathrm{kDa}$ glycoprotein synthetized in the liver and normally circulates in plasma at $160-400 \mathrm{mg} / \mathrm{dl}$ [2]. It is composed of two sets of three different polypeptides chains $(\mathrm{A} \alpha, \mathrm{B} \beta, \gamma)_{2}$, arranged in three nodules: the $\mathrm{N}$ terminal of the six chains converge at the center forming the globular E region. A coiled coil of all the three chains extends from each side of the $\mathrm{E}$ domain to connect with the outer D domains, which form from the C-terminal region of the $B \beta$ and $\gamma$ chains. The coiled coil is delineated by two disulfide rings and its central region has a kink in 
its structure that acts as the primary attack site for plasmin [3].

Inherited fibrinogen disorders can be quantitative (Type I; absence or decreased level of circulating fibrinogen, afibrinogenemia and hypofibrinogenemia, respectively) or qualitative (Type 2; normal or decreased antigenic levels and low fibrinogen activity, dysfibrinogenemia and hypodysfibrinogenemia, respectively) [2, 4].

Dysfibrinogenemia is caused by structural abnormalities that can be inherited (congenital) or acquired [5]. Inherited dysfibrinogenemia is caused by mutations in the coding region of the fibrinogen $A \alpha, B \beta$ or $\gamma$ genes and the majority of cases result from heterozygous missense mutations [4]. The prevalence of inherited dysfibrinogenemia in the general population is unknown [5]. The pattern of inheritance is autosomal dominant, and $55 \%$ of the patients are asymptomatic, while $25 \%$ develop bleeding or thrombosis. Hypodysfibrinogenemia has features of both hypo- and dysfibrinogenemia: the reduced circulating fibrinogen levels confers a hypofibrinogenemia phenotype, and the expression of the mutation that alters functionality, a dysfibrinogenemic phenotype [6]. As with dysfibrinogenemia, in hypodysfibrinogenaemia bleeding extends from mild to moderate, but individuals are more predisposed to thrombosis.

The diagnosis of qualitative fibrinogen disorders is done by the measurement of standard clotting times, whose sensitivity depends upon the methods, reagents and coagulometers used [4]. Usually the thrombin time is prolonged, although with some variants it may be normal [5].

During fibrinogen synthesis, each newly synthetized chain is independently translocated into the endoplasmic reticulum (ER) where chaperones assist in the assembly and folding processes. Molecules are assembled in a step wise manner in the lumen of the ER: first two-chain $A \alpha-\gamma$ and $B \beta-\gamma$ complex are formed. These complexes recruit a $B \beta$ or $\mathrm{A} \alpha$ chain respectively and form half-molecules $(\mathrm{A} \alpha-\mathrm{B} \beta-\gamma)_{1}$, which in the last step dimerize through $\mathrm{N}$-terminal disulphide bridges to form $(A \alpha-B \beta-\gamma)_{2}$ hexamers [7]. Several studies performed in recombinant systems, using deletion and substitution mutants, indicate that an intact coiled-coil and correct inter- and intrachain disulphide bonds are needed for final molecular assembly [8-10]. In afibrinogenemia mutated molecules are usually absent from circulation. However, in hypofibrinogenemia or hypodysfibrinogenemia if the mutation impairs assembly variant molecules may be secreted, but underrepresented in plasma.

Here we describe a new variant with an $\mathrm{A} \alpha$ Arg104 $\rightarrow$ Cys mutation in the coiled-coil region that we have named fibrinogen Caracas IX.

\section{Methods}

\section{Materials}

Bovine thrombin was from Enzyme Research Laboratories (South Bend, IN). Lysine-sepharose 4B was from GE
Healthcare (Piscataway, NJ). Pefabloc ${ }^{\circ}$ SC (4- (2-Aminoethyl) benzenesulfonyl fluoride hydrochloride) was from Fluka, Sigma-Aldrich (Buchs, Switzerland). Tissuetype plasminogen activator and plasmin were from American Diagnostica (Stamford, CT). Human albumin and benzamidine were from Sigma Chemical Company (St Louis, MO). Albumin antibody conjugated to peroxidase was from Dako Corporation (Carpinteria, CA). The substrate 3,3'-diaminobenzidine (DAB) was from Thermo Scientific (Rockford, IL). The LabTek chambers and Alexa Fluor 488 were purchased from Invitrogen, Nalge Nunc International (Rochester, NY).

\section{Blood collection}

Blood was collected in citrate ( 1 volume of $0.13 \mathrm{M}$ trisodium citrate and 9 volumes of blood), the first $3 \mathrm{ml}$ of blood discarded, and centrifuged twice at $2000 \mathrm{~g}$ for $10 \mathrm{~min}$. The platelet poor plasma obtained (PPP) was supplemented with benzamidine $10 \mathrm{mM}$ (final concentration), except the plasma to be used for fibrinolysis experiments, aliquoted and kept at $-80{ }^{\circ} \mathrm{C}$ until use. Routine coagulation tests were performed with citrated plasma on coagulation analyzer STA Compact ${ }^{\circ}$, Stago, France. Fibrinogen level was determined by Clauss (Laboratoire Stago, Asnière, France) and clot weight method [11]. Antigenic fibrinogen concentration was measured by a latex immunoassay (Liaphen Fibrinogen, Hyphen BioMed, France).

\section{Mutation analysis}

Blood was collected in $0.5 \mathrm{M}$ ethylenediaminetetraacetate tetrasodium salt (EDTA $\left.\mathrm{Na}_{4}\right)(50: 1)$. Genomic DNA was isolated using the Invisorb Spin Blood Mini Kit (Invitek GmbH, Berlin, Germany) according to the manufacturer's protocol. Sequences comprising all exons and exon-intron boundaries from the three fibrinogen genes: FGA, FGB, and FGG were amplified by the polymerase chain reaction (PCR) according to standard protocols. After purification of the PCR products using the Invisorb Spin PCRapid Kit (Invitek, Berlin, Germany), direct DNA cycle sequencing was performed, applying the Big Dye kit from Applied Biosystems (Foster City, CA), according to the manufacturer's recommendations.

\section{Fibrinogen purification}

Fibrinogen was purified essentially as described elsewhere with modifications [12]. Plasma samples were thawed and supplemented with $1 \mathrm{mM}$ Pefabloc $^{\circ}$ and 5 mM EDTA (final concentrations). Plasma was depleted of plasminogen by passing through a lysine-sepharose $4 \mathrm{~B}$ column, and then fibrinogen was purified by precipitation $(\times 3)$ with $25 \%$-saturation ammonium sulphate, $\mathrm{pH}$ 7.5. This fibrinogen fraction also contained copurifying fibronectin, factor XIII and $\mathrm{vW}$ factor. The 
precipitate was dissolved in $0.3 \mathrm{M} \mathrm{NaCl}$, dialyzed against the same solution, and stored at $-80{ }^{\circ} \mathrm{C}$ until used.

The integrity of the purified fibrinogen was analyzed by sodium dodecylsulfate-polyacilamide gel electrophoresis (SDS-PAGE) on $8 \%$ gel. The coagulability of the purified fibrinogen was $96 \%$ and $93 \%$ and the yield 43 and $27 \%$, control and patient, respectively.

\section{Fibrinogen degradation}

Fibrinogen was incubated with plasmin as described [13] with minor modifications. Purified fibrinogen $(0.9 \mathrm{mg} /$ $\mathrm{ml}$, in TBS) was incubated with plasmin $(18 \mu \mathrm{g} / \mathrm{ml}$, in TBS) in the presence of $1 \mathrm{mM} \mathrm{CaCl}$ or $5 \mathrm{mM}$ EDTA at $37{ }^{\circ} \mathrm{C}$ at different incubation times $(15,30 \mathrm{~min}$ and $4 \mathrm{~h}$ ), quenched with $2 \%$ SDS-DTT (v:v) sample buffer and immediately boiled. The zero time sample contained no plasmin. The fibrinogen degradation products were analyzed in a $6 \%$ gel SDS-PAGE under non reducing conditions.

\section{Western blotting}

In order to detect fibrinogen-albumin complexes, Western blotting was performed under non reduced conditions essentially as described [14]. Briefly, purified fibrinogens $(5 \mu \mathrm{g})$ and human albumin $(5 \mu \mathrm{g})$ were loaded in a $5 \%$ gel SDS-PAGE, and electroblotted onto nitrocellulose [15]. The membrane was incubated for $2 \mathrm{~h}$ with anti-human albumin antibody conjugated to peroxidase (1:1000). The cross-reacting bands were detected with $0.6 \% 3$, 3'diaminobenzidine (DAB), $3 \%$ cobalt chloride and $3 \%$ hydrogen peroxide.

\section{Activated factor XIII (FXIIla) fibrin cross-linking}

The kinetics of fibrin cross-linking was examined essentially as described elsewhere [14]. Fibrin was crosslinked by the endogenous factor XIIIa that precipitated together with fibrinogen during the purification process. Purified fibrinogen $(1 \mathrm{mg} / \mathrm{ml})$ was clotted with $1 \mathrm{U} / \mathrm{ml}$ of thrombin and $5 \mathrm{mM} \mathrm{CaCl} \mathrm{C}_{2}$. The reactions were quenched at different time points $(0,2,5,15 \mathrm{~min}$ and 1 , $4,24 \mathrm{~h}$ ) with $2 \%$ SDS-DTT and analyzed in a $8 \%$ gel SDS-PAGE.

\section{Fibrin polymerization}

The kinetics of fibrin formation were studied in plasma and purified fibrinogen [16]. Briefly, $100 \mu \mathrm{l}$ fresh plasma or $0.5 \mathrm{mg} / \mathrm{ml}$ purified fibrinogen in $50 \mathrm{mM}$ Tris, $0.15 \mathrm{M}$ $\mathrm{NaCl}, \mathrm{pH} 7.4$ (TBS) were dispensed in a 96-well plate. Then $10 \mu \mathrm{l}$ of $1 \mathrm{unit} / \mathrm{ml}$ bovine thrombin - $20 \mathrm{mM} \mathrm{CaCl}_{2}$ (final) was added to plasma or 5 units $/ \mathrm{ml}$ bovine thrombin and $5 \mathrm{mM} \mathrm{CaCl}_{2}$ to fibrinogen solution. The changes in optical density (OD) were recorded every $15 \mathrm{~s}$ over $1 \mathrm{~h}$ at $350 \mathrm{~nm}$ in a Tecan Infinite $\mathrm{e}^{\bullet}$ 200. The polymerizations were done in three different experiments in triplicate. The lag time $(\mathrm{s})$, slope $(\mathrm{mOD} / \mathrm{s})$ and final turbidity (mOD) were calculated from each curve and averaged.

\section{Fibrinolysis}

The method was performed as described by Carter et at 2007 [17] with minor modifications. The PPP aliquots without benzamidine were used. Twenty five $\mu$ l of PPP were aliquoted in a 96-well plate, then $75 \mu \mathrm{l}$ of tPA $166 \mathrm{ng} / \mathrm{ml}$ diluted in $20 \mathrm{mM}$ Hepes, $0.15 \mathrm{M} \mathrm{NaCl}$, $\mathrm{pH} 7.4$ was added. Clotting was initiated by adding $50 \mu \mathrm{l}$ of thrombin- $\mathrm{CaCl}_{2}(0.03 \mathrm{U} / \mathrm{ml}$ and $9 \mathrm{mM}$, respectively).The OD changes were recorded at $350 \mathrm{~nm}$ every $15 \mathrm{~s}$ over $1.5 \mathrm{~h}$ in a TECAN ${ }^{\odot}$ infinite $2 \mathrm{M}$ microplate reader. The fibrinolysis was done at least three times in triplicate. The time to degrade $50 \%$ of the clot (T50\%) was calculated from the time elapsed from half the value of the maximum absorbance of the polymerization to halfthe value decreased of the maximum absorbance of the lysis curve branch. The rate of clot degradation (slope) was calculated in the descending part of the curve and the absolute value reported.

\section{Direct plasma mass spectrometry}

Plasma was precipitated with saturated $\left(\mathrm{NH}_{4}\right)_{2} \mathrm{SO}_{4}(25 \%$, final), and the precipitate washed $(2 \times)$ with $25 \%$ saturated $\left(\mathrm{NH}_{4}\right)_{2} \mathrm{SO}_{4}$. The pellet was dissolved in $8 \mathrm{M}$ urea, $30 \mathrm{mM}$ dithiothreitol, $50 \mathrm{mM}$ Tris- $\mathrm{HCl}, \mathrm{pH} 8.0$ and left $3 \mathrm{~h}$ at $37{ }^{\circ} \mathrm{C}$. The reduced sample was injected into an Agilent 6230 Accurate-Mass electrospray time-of-flight (TOF) mass spectrometry system [18]. A Poroshell 300SB C3 $(2.1 \times 75 \mathrm{~mm})$ column was used with an acetonitrile gradient and profile data was collected. Multi charged spectral envelopes were deconvoluted using maximum entropy processing and BioConfirm software with an isotope width of $15 \mathrm{Da}$.

\section{Clots biophysical characterization}

In order to characterize some biophysical parameters of the clot structure, the elastic modulus, the Darcy constant (Ks), and fibrin network imaging by confocal microscopy were performed. For these experiments a healthy man with plasma fibrinogen concentration similar to the patient was chosen as a control.

\section{Elastic modulus}

The fibrin elastic modulus (EM) was measured in the

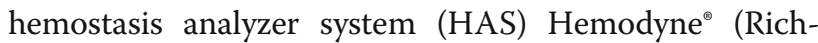
mond, VA). Briefly, $700 \mu \mathrm{L}$ of plasma was placed in the plastic cone and incubated for $1 \mathrm{~min}$ at $37{ }^{\circ} \mathrm{C}$. Then $50 \mu \mathrm{L}$ of a thrombin $-\mathrm{CaCl}_{2}$ solution $(1.3 \mathrm{U} / \mathrm{ml}$ and $25 \mathrm{mM}$ final concentrations; respectively) was gently and carefully mixed in. The increase in the EM was recorded every 1 min over a $30 \mathrm{~min}$ period. Each sample was run in triplicate in three independent experiments. The EM 
(kdyne $/ \mathrm{cm}^{2}$ ) reported corresponds to the averaged EM value that was reached at $30 \mathrm{~min}$.

\section{Permeation}

Permeation through plasma clots was recorded essentially as described elsewhere [19]. The clotting conditions used were $1 \mathrm{U} / \mathrm{ml}$ of thrombin and $20 \mathrm{mM} \mathrm{CaCl}$ (final concentrations). The clots were left for $2 \mathrm{~h}$ in a moist environment at $37^{\circ} \mathrm{C}$ in order to fully polymerize. The buffer percolated through the columns was TBS. Nine clots of each sample were run per experiment $(n=3)$ and one measurement per clot was taken.

The Darcy constant (Ks) was calculated using the following equation [20]:

$$
\mathrm{Ks}=\mathrm{QL \eta} / \mathrm{tAP}
$$

Where $\mathrm{Q}=$ volume of the buffer $\left(\right.$ in $\mathrm{cm}^{3}$ ), having a viscosity $\eta$ of 0.01 (poise), flowing through the column of height $\mathrm{L}(\mathrm{cm})$ and area $\mathrm{A}\left(\mathrm{cm}^{2}\right)$ in a given time (s) under a hydrostatic pressure $\mathrm{P}\left(\right.$ dyne $\left./ \mathrm{cm}^{2}\right)$.

\section{Confocal microscopy}

The experiments were done essentially as described elsewhere [16]. Briefly, clots were formed inside the eight wells LabTek chambers (Invitrogen, Nalge Nunc International, Rochester, NY). Plasma samples were mixed with Alexa Fluor 488-labeled fibrinogen $(10 \mu \mathrm{g} / 315 \mu \mathrm{l}$ final sample volume), then clotted with thrombin- $\mathrm{CaCl}_{2}$ solution $(0.3 \mathrm{U} / \mathrm{ml}$ and $20 \mathrm{mM}$, respectively, final concentration). The clots were left for $2 \mathrm{~h}$ in a moist environment at $37^{\circ} \mathrm{C}$ in order to fully polymerize.

The fibrin clots were observed in a Nikon Eclipse TE 2000 U laser scanning confocal microscopy (LSCM), with an argon ion laser (473 $\mathrm{nm}$ excitation and 520/ $540 \mathrm{~nm}$ for emission). The objective used was Plan APO VC 60X water immersion with a work distance of 0.27 . The acquisition pinhole was set to $60 \mu \mathrm{m}$. Image analyses were done as described [21]. A z-stack of 60 slice was use for construct a $3 \mathrm{D}$ projection of $30 \mu \mathrm{m}$ thick (0.5 $\mathrm{\mu m} / \mathrm{slice})$ were done. Five image by clot $(212 \times 212 \mu \mathrm{m})$ for each experiment (control and patient) were accomplished. Two diagonal lines, a horizontal and a vertical were drawn on the volumetric image of the stack using the Olympus FV10-ASW 2.1 software for obtain the pseudocolor perfil by line. Line graphs were used to calculate density (picks/ $\mu$ ) and diameter of fibers $(\mu \mathrm{m})$ with Origin Pro 8 software.

\section{Dynamic fibrin clot growth}

The spatio temporal dynamics of fibrin clot formation in real time was assessed in plasma by measuring light scattering over 30 min every $15 \mathrm{~s}$ using a Thrombodynamics Analyser System (HemaCore, Moscow, Russia) as previously described [21]. Briefly, plasma coagulation is activated when it is brought in contact with tissue factor coated on a plastic cuvette. The clot formation begins on the activator and propagates into the bulk of plasma in which no TF is present. Images are analyzed computationally to measure lag time, initial and stationary growth rate, size at $30 \mathrm{~min}$ and clot density. Based on the plots of clot size versus time, the initial velocity of clot growth is measured as the mean slope over the 2-6 min period (characterizing the VIIa-TF pathway) and the stationary velocity of clot growth is measured as the mean slope over the 15-25 min period [22].

\section{Results}

Case report

A new abnormal fibrinogen was discovered accidentally in an asymptomatic 11-year-old girl when routine preoperative coagulation tests were performed for nasal turbinate surgery, which proceeded successfully. Her parents reported that when she was 3 years old she had surgery for stenosing tenosynovitis without any complications. Haemostasis work-up showed that the thrombin time was marginally prolonged, and that functional and antigenic fibrinogen levels were decreased without discrepancy in the proband and her father (Table 1). Neither parent report any hemostatic problems. Full DNA sequencing of $F G A, F G B$ and FGG revealed that proband was heterozygous for a novel point mutation in FGA g. $3057 \mathrm{C}>\mathrm{T}$ that gives rise to an A $\alpha$ Arg104 > Cys substitution (numbered without the signal peptide). In addition, she was found to be heterozygous for the $A \alpha$ Ala312/Thr polymorphism. Targeted sequencing of the father showed he was also heterozygous for the novel A $\alpha$ Arg104 > Cys mutation but his A $\alpha$ Ala312/Thr status was not explored.

Table 1 Summary of coagulation tests

\begin{tabular}{llll}
\hline & Proband & Father & Reference values \\
\hline$\pi$ (s) & 18.6 & 30.8 & $16-18$ \\
PT (s) & 15.8 & 54.6 & $13-15$ \\
aPTT (s) & 34.6 & 42.2 & $27-34$ \\
Fibrinogen (Clauss) & 163 & 122 & \\
(mg/dl) & 151 & & $200-400$ \\
& 158 & & \\
Fibrinogen antigen (mg/dl) & 177 & 126 & $190-400$ \\
Factor V (\%) & 79 & - & $60-140$ \\
Factor II (\%) & 105 & 88 & $60-140$ \\
Factor VII (\%) & 79 & 69 & $65-145$ \\
Factor X (\%) & 82 & 67 & $65-130$ \\
\hline
\end{tabular}

$\pi$ thrombin time, $P T$ prothrombin time, aPTT activated partial thromboplastin time -: not done 


\section{Plasma mass spectrometry}

Electrospray TOF MS of purified fibrinogen from the proband and her father showed normal masses and isoforms for the $B \beta$ and $\gamma$ chain components, with no evidence of any mutations (not shown). Examination of extracted A $\alpha$ chain spectra from a control, who was homozygous for the A $\alpha$ Thr312 polymorphism, showed the expected major peak at 66,136 Da corresponding to the non-phosphorylated form of the A $\alpha$ chain (theoretical mass 66,132 Da) (Fig. 1). With a peak at 66,134 Da the father appeared to have one normal copy of the A $\alpha$ Thr312 allele and one variant copy giving rise to a protein of mass $66,080 \mathrm{Da}$. This mass decrease of $54 \mathrm{Da}$ was entirely consistent with a point mutation of Arg $\rightarrow$ Cys $(-53 \mathrm{Da})$. Spectra shows the proband inherited this same variant (theoretical mass $66,079 \mathrm{Da}$ ) from her father, together with a copy of the less common A $\alpha$ Ala312 allele from her mother. Interestingly the variant chain with the Arg $\rightarrow$ Cys substitution was underrepresented in plasma fibrinogen and contributed only about $25 \%$ of the total A $\alpha$ chain material.

Hereafter, all the studies performed to characterize the new abnormal fibrinogen were performed with the plasma of the proband's father (indicated as patient), except the fibrin polymerisation and the fibrin clot growth assessment performed in both proband and proband's father.

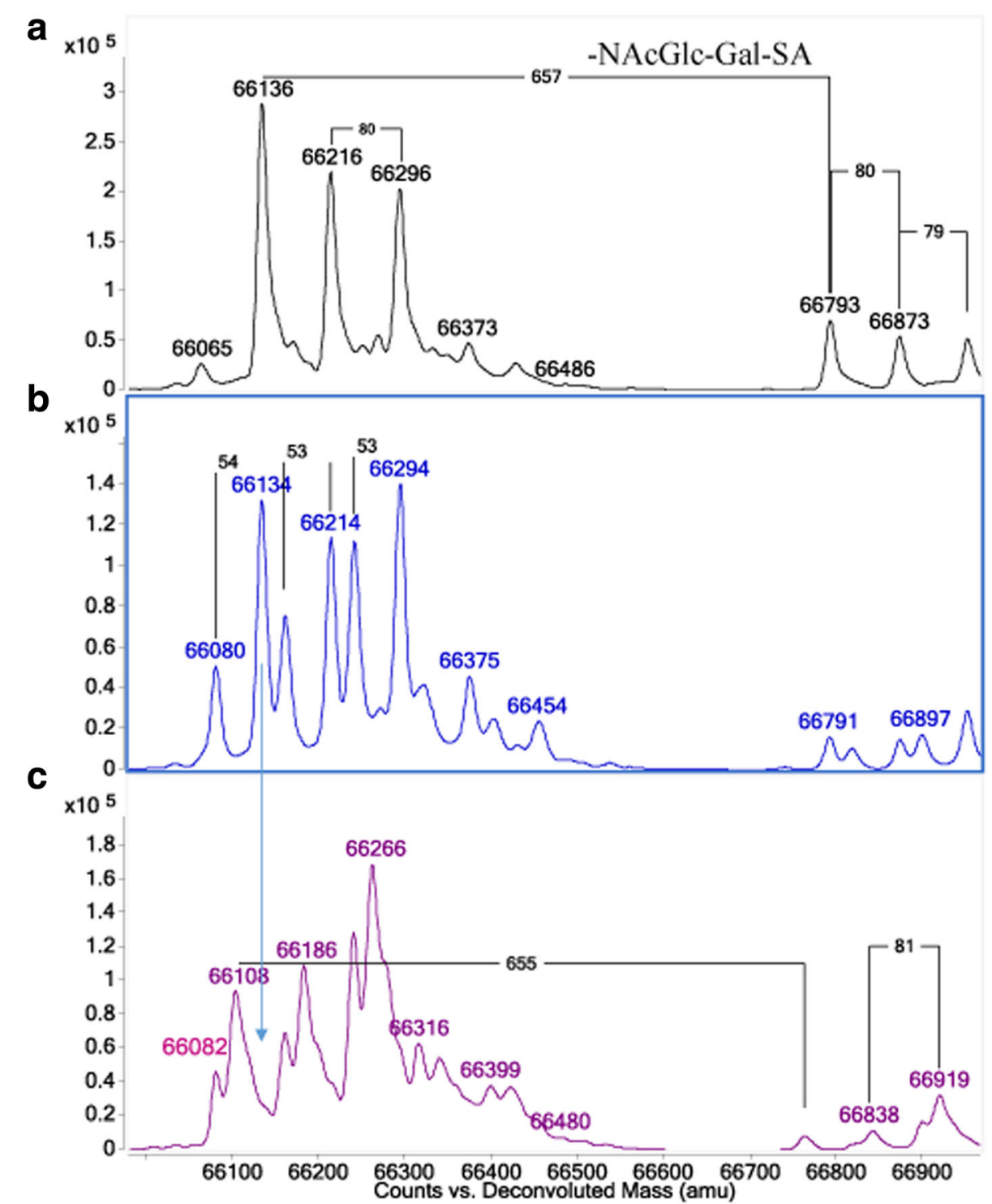

Fig. 1 Transformed electrospray TOF spectra of fibrinogen Aa chains. a Normal control homozygote for the Aa Thr312 allele, (b) father, (c) proband. The father showed a normal Aa312Thr chain at 66,134 Da with a new variant chain at 66,080 Da containing an Arg $\rightarrow$ Cys mutation (-53 Da). The proband, who had no normal Aa312Thr chains, was heterozygous for the new variant and an Aa312Ala chain (66,108 Da) inherited from her mother. Peaks at +80 Da reflect successive Ser-phosphorylation. The $Y$ - axis depicts a relative voltage response in arbitrary units 
Fibrinogen degradation, western blotting and fibrin factor XIIla cross-linking

Plasmin attacks the middle of the coiled-coil of fibrinogen and generates the degradation products fragments $Y$ and D. Surprisingly, the degradation of patient fibrinogen (A $\alpha$ Arg104 > Cys) by plasmin was similar to control in the presence of either $\mathrm{Ca}^{2+}$ or EDTA (results not shown). In addition, the mutation introduces an unpaired - $\mathrm{SH}$ that could potentially form fibrinogenalbumin complexes, although by immunoblotting fibrinogen binding to albumin was negative (results not shown). The patient fibrin $\alpha$-chain factor XIIIa crosslinking seemed faster compared to control. It can be seen in Fig. 2 that the patient has more intense bands of higher molecular weight corresponding to $\alpha$-chain factor XIIIa cross-linking at short incubation times (i.e. 2, 5, and $15 \mathrm{~min}$ ) compared to control.

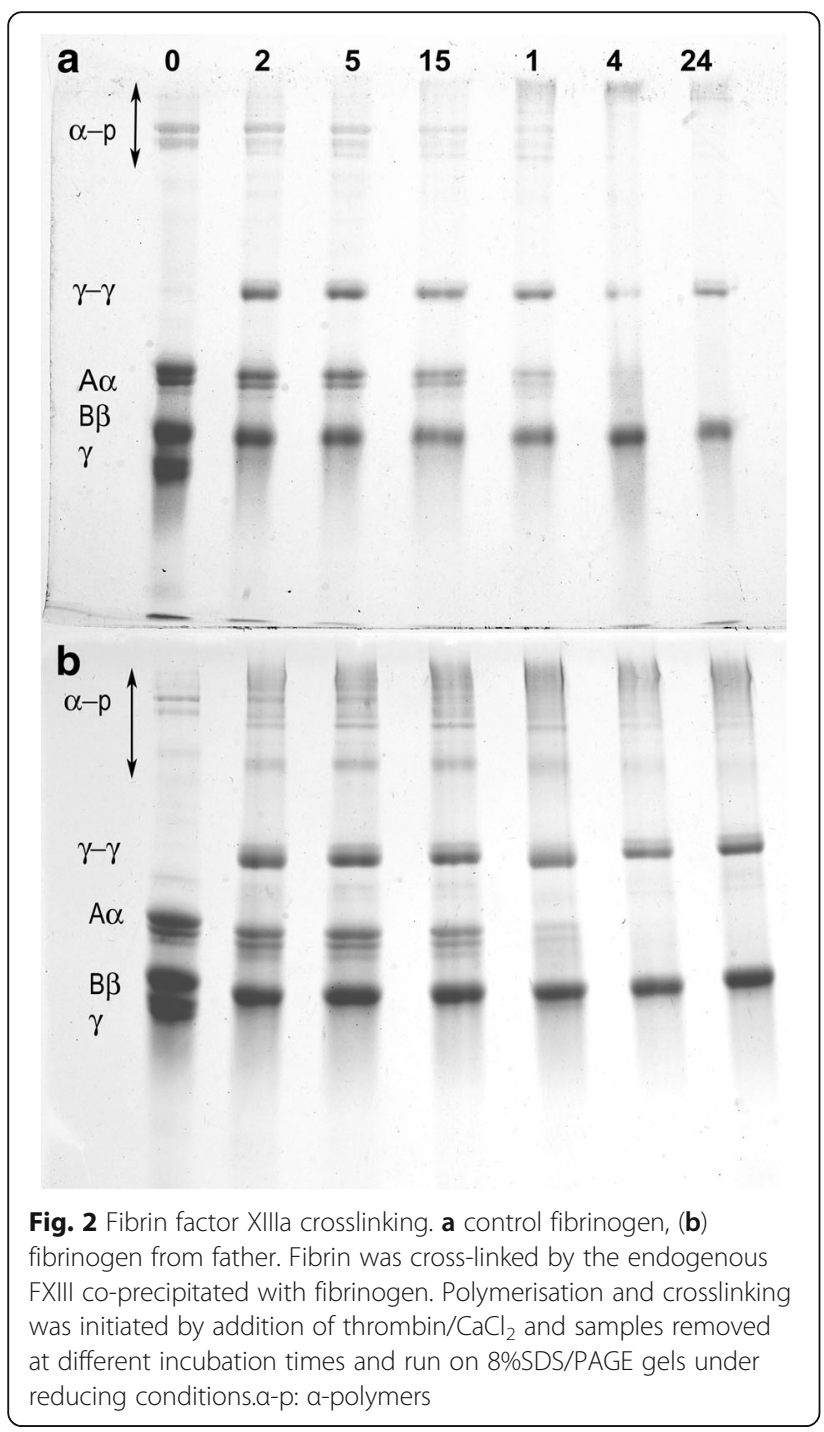

Table 2 Plasma fibrin polymerization. Plasma polymerization was done with fresh plasma. The optical density (OD) was multiplied by 1000 (mOD). The results are presented as mean $( \pm S D)$

\begin{tabular}{lllll}
\hline Parameters & Control & Proband & Father & Mother \\
\hline Fg* $^{*}[\mathrm{mg} / \mathrm{dl}]$ & 258 & 183 & 168 & 523 \\
Lag time $(\mathrm{s})$ & 0 & 0 & $7.5 \pm 11$ & 0 \\
Slope $(\mathrm{mOD} / \mathrm{s})$ & $2.23 \pm 0.19$ & $1.66 \pm 0.28$ & $0.86 \pm 0.24$ & $2.38 \pm 0.58$ \\
MaxAbs (mOD) & $833 \pm 18$ & $736 \pm 0.6$ & $520 \pm 4$ & $970 \pm 31$
\end{tabular}

* Fg: fibrinogen, by clot weight method [11]

MaxAbs maximum absorbance

\section{Fibrin polymerization and Fibrinolysis}

Fibrin formation in the proband's plasma had a near normal profile with only a slightly decreased final turbidity $(\sim 12 \%)$, while in the father the fibrin fibers growth (reflected in the slope value) and consequently the final turbidity was decreased approximately $1.3 \times$ and $40 \%$, respectively (Table 2, Fig. 3a). With purified fibrinogen,

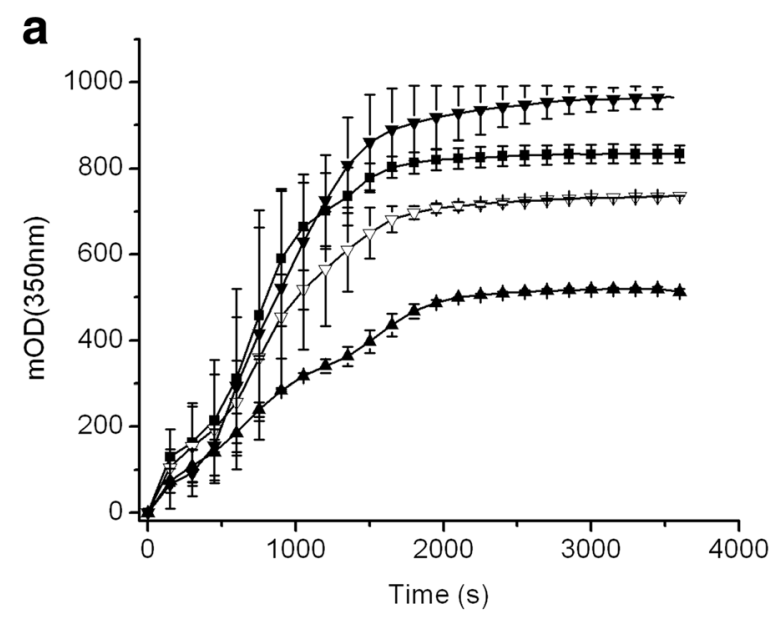

b

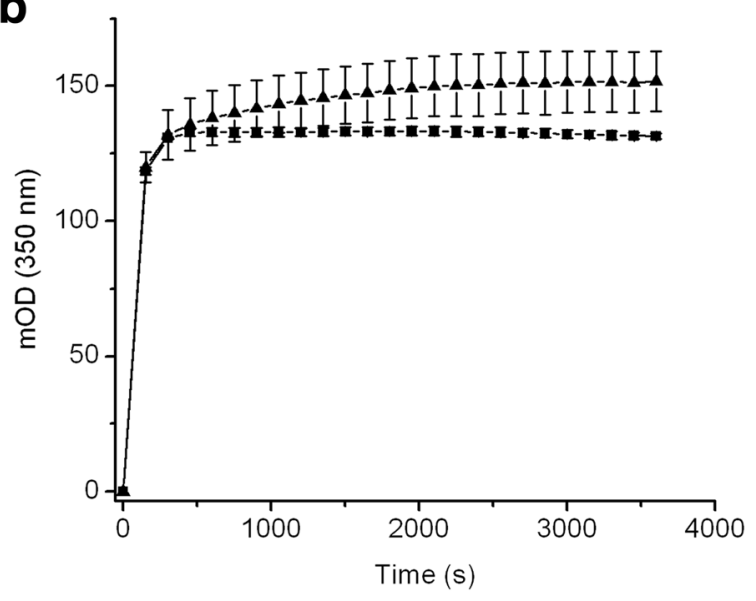

Fig. 3 Fibrin polymerization curves. a fresh plasma, (b) purified fibrinogen. $\mathbf{m}$ : control, $\mathbf{\nabla}$ : mother, $\nabla$ : proband, $\mathbf{\Delta}$ : father 
the proband's father polymerization was similar to control (Fig. 3b).

The father's fibrin clot dissolution had a slightly shorter T50: $485 \pm 33 \mathrm{~s}$ compared to $613 \pm 62 \mathrm{~s}$ in control $(p=0.001)$, and a slightly delayed fibrinolysis rate: $1.9 \pm 0.4 \times 10^{-4} \mathrm{OD} / \mathrm{s}$ compared to $2.5 \pm 0.4 \times 10^{-4}$ $\mathrm{OD} / \mathrm{s}$ in control $(p=0.008)$. In Fig. $4 \mathrm{a}$ are shown the fibrinolysis curves, the patient area under the curve (AUC) was $1.54 \times 10^{7}$ compared to $3.64 \times 10^{7}$ control, approximately $2 \times$ difference, and in Fig. $4 \mathrm{~b}$ the T50 s and slopes distribution are represented.

\section{Clots biophysical characterization}

The patient fibrin elastic modulus was approximately 500 dyne $/ \mathrm{cm}^{2}$ less than the control, but this was not statistical significant (Table 3 ). The patient clot's surface available for flux (Ks) was almost $1.8 \times$ higher than control ( $\mathrm{p}<<0.001)$. The confocal microscopy images showed subtle differences between the patient fibrin network and control (Fig. 5). The patient fibrin density and diameter were $0.329 \pm 0.016$ peaks $/ \mu \mathrm{m}$ and $1.150 \pm 0.642 \mu \mathrm{m}$, respectively, compared to 0.316 peaks/ $\mu \mathrm{m} \pm 0.017$ and $1.23 \pm 0.02 \mu \mathrm{m}$ in the control $(p<0.05)$. In the orthogonal fibrin clot view the patient clot looked more porous, which correlated with the porosity value $(\mathrm{Ks})$ that was approximately $2 \times$ higher.

\section{Thrombodynamics}

As shown in Table 4, all the values of thrombodynamics parameters were decreased in the proband and her father compared to the healthy control. The lag time, the initial rate and the clot size at 30 min were similar between the proband and her father. The clot density was lower in father compared to the proband $(9625.5$ versus 11,206 arbitrary unit) reflecting his lower fibrinogen concentration. Videos of dynamic clots growth are
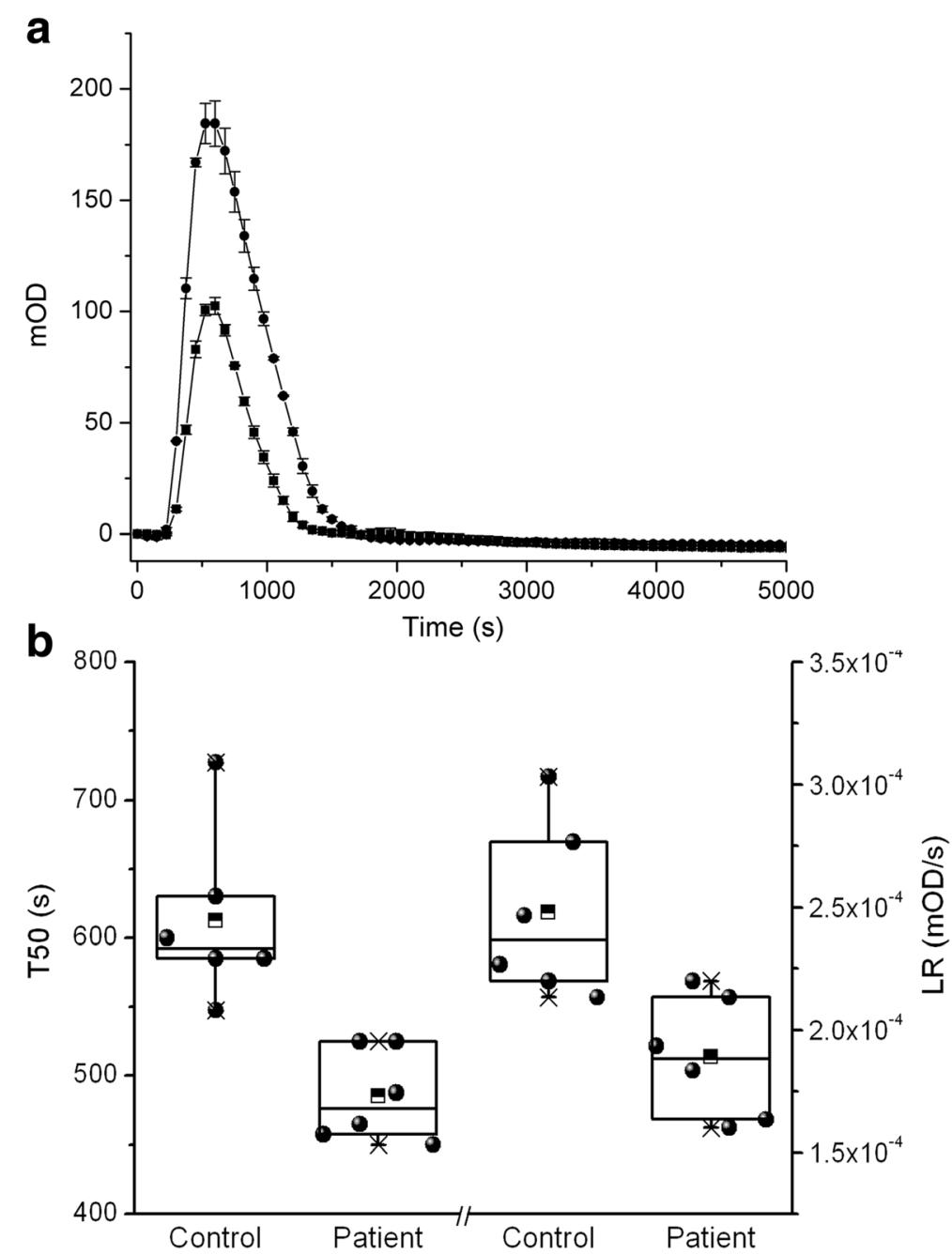

Fig. 4 a Fibrinolytic process induced by t-PA. Control (•), father ( $\mathbf{(})$. The fathers clot was completely dissolved earlier than control. b Box chart of the T50 and lysis rate (LR) 
Table 3 Summary of clots biophysical characterization. Results are showed as the mean $( \pm S D)$. In brackets the number of values averaged

\begin{tabular}{llll}
\hline & Control & Father & $p$ \\
\hline Fg $(\mathrm{mg} / \mathrm{dl})$ & 222 & 176 & \\
EM $\left(\right.$ dyne $\left./ \mathrm{cm}^{2}\right)$ & $\begin{array}{l}3239 \pm 671 \\
(n=9)\end{array}$ & $\begin{array}{l}2734 \pm 534 \\
(n=7)\end{array}$ & 0.116 \\
& $\begin{array}{l}5.1 \pm 0.9 \\
\text { Ks } \times 10^{-9}\end{array}$ & $\begin{array}{l}9.4 \pm 1 \\
(n=20)\end{array}$ & $1.83 \times 10^{-17}$ \\
$\left(\mathrm{~cm}^{2}\right)$ & $(n=22)$ & \\
\hline
\end{tabular}

Fg: fibrinogen concentration by clot weight method. EM: elastic modulus. Ks: permeation constant

shown in Additional file 1 (control), Additional file 2 (father) and Additional file 3 (proband).

\section{Discussion}

A new fibrinogen mutation was found accidentally in an 11 year-old girl when routine pre-operative coagulation tests were performed. Gene sequencing revealed a missense mutation in FGA: g. $3057 \mathrm{C}>\mathrm{T}$ that predicts a p. Arg104 > Cys substitution. The low functional and antigenic fibrinogen concentrations in the father and daughter can be explained by the low expression level of the A $\alpha$ Arg104 $\rightarrow$ Cys chain in their plasma fibrinogen as the new variant made up only about $25 \%$ of the total. Also, because the A $\alpha$ Arg104 $\rightarrow$ Cys mutation creates a potential N-glycosylation site (Asn-Asn-Cys) centered on Asn103, mass specters were carefully examined for any new $A \alpha$ chains with bi-antennary oligosaccharide side chains, but none were observed at or around their expected position at +2202 Da.

The fibrinogen coiled-coil connects the central E nodule with the distal nodule $\mathrm{D}$ and is made up of $\mathrm{A} \alpha$ chain
Table 4 Thrombodynamics data

\begin{tabular}{llll}
\hline & Control & Proband & Father \\
\hline Lag time $(\mathrm{min})$ & 1.1 & 1.45 & 1.4 \\
Initial rate $(\mu \mathrm{m} / \mathrm{min})$ & 57.45 & 57.5 & 63.3 \\
Stationary rate $(\mu \mathrm{m} / \mathrm{min})$ & 56.4 & 32.1 & 33.7 \\
Size at $30 \mathrm{~min}(\mu \mathrm{m})$ & 1814 & 1306.5 & 1368.6 \\
Density (a.u.) & 22,161 & 11,026 & 9626.5 \\
\hline Min minutes, au. arbitrary unit & & &
\end{tabular}

residues 50-160, B $\beta$ 81-191 and $\gamma$ 24-134 [3]. At the middle of the coiled coil the structure become disorganized, probably due to the presence of proline residues at $\gamma 70$ and 76 [3]. There are 19 afibrinogenemia, 6 hypofibrinogenemia and 2 dysfibrinogenemia mutations reported in the stretch of A $\alpha$ 100-112 (http:// www.geht.org). At present, there are only 3 hypodysfibrinogenemia reported in the middle of the coiled coil region: Fibrinogen Epsom (B $\beta$ Asn137_Glu141), Michigan ( $\gamma$ Tyr114His), and Leipzig II (Ala82Gly) [6], although the last two are compound heterozygous. These naturally occurring fibrinogen variants confirm previous findings about the role of the helical coiled coil in fibrinogen biosynthesis and secretion [10]. Other mutations in this region cause dysfibrinogenemia. For example, fibrinogen Plzen (A $\alpha$ Asn106Asp) and Vizovice (A $\alpha$ Phe98Ile) are characterized by low functional fibrinogen, $1.13 \mathrm{~g} / \mathrm{l}$ and 1.66 , but normal immunologic fibrinogen concentration $3.99 \mathrm{~g} / \mathrm{l}$ and 2.89 , respectively $[23,24]$. The normal fibrinogen antigenic concentration excludes that these abnormal fibrinogens have impaired secretion. The low fibrinogen activity highlight the role of this part of the molecule on fibrinogen polymerization.
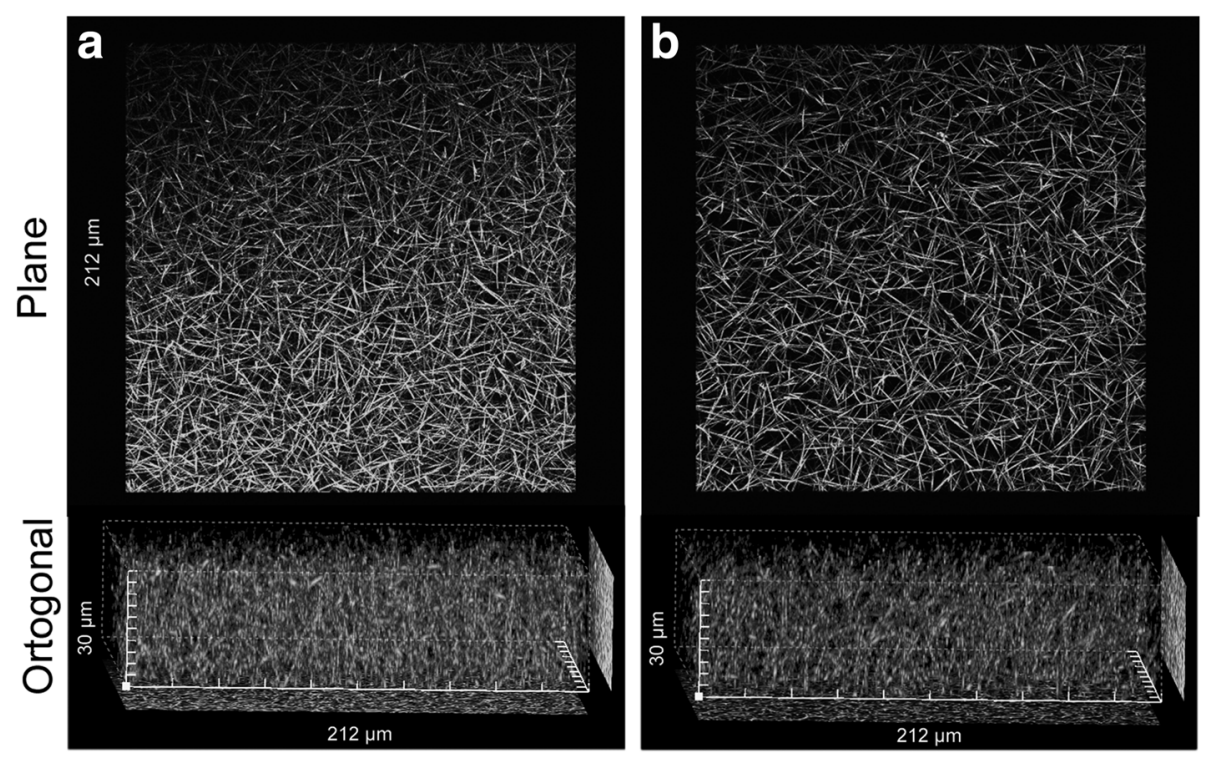

Fig. 5 Confocal microscopy images of plasma clots. a Control, (b) Father. The fibrin was labeled with Alexa488 coupled to fibrinogen 
The kinetics of fibrin formation in plasma studied by turbidity was more impaired in the father compared to the proband. However, by thrombodynamics the father and proband behaved similarly. The results obtained using this last technique seemed more consistent with mass spectrometry. Similar polymerization kinetic would be expected for both individuals as difference of $0.15 \mathrm{~g} / \mathrm{l}$ in their functional fibrinogen concentrations appeared insufficient to cause major changes polymerization. These discrepant results could result from the fact that the thrombodynamics uses a primarily different principle to initiate the coagulation cascade compared to the turbidity assay. In the former, the clotting is activated by a surface with immobilized tissue factor, which better resembles in vivo clot formation, whereas in the latter the clotting is activated by homogeneously dissolved thrombin. Fibrinogen functional correlations between these two assays require further studies.

We also expected the father would exhibit altered fibrin formation in a purified system, but probably the mutated molecules were underrepresented compared to plasma. In addition, we do not know if the mutated molecules were lost during the purification process. In the future it would be advisable for these cases to choose an immunopurification method.

The proband had higher fibrinogen concentration than her father, despite being about 5 decades younger. Different studies have found that fibrinogen increases with age [25-27]. Hager et al. reported a $25 \mathrm{mg} / \mathrm{dl}$ increases per decade [26]. Since fibrinogen is an acute phase reactant protein and CRP was not measured, it cannot be ruled out that the proband at the time of blood withdrawal could have an infectious disease.

When analyzed, an hypofibrinolysis has been observed in most hypodysfibrinogenemia [6]. The A $\alpha$ Arg104 and B $\beta$ Lys133 are the very first plasmin attack points. However, the fibrinolytic process of fibrinogen Caracas IX was close to normal. In contrast, fibrinogen Epsom with a deletion of the residues Asn137_Glu141 showed hyperfibrinolysis, together with an increased fibrinogen clearance [28]. In fibrinogen Dunedin whose Y82Ala $\rightarrow$ Gly mutation occurs near the plasmin sensitive site in the coiled coil region also displayed an increased proteolytic sensitivity. It would be interesting in the future to explore the causes of these differences in order to shed light on possibly unknown mechanisms of fibrin(o)gen degradation.

\section{Conclusions}

The fibrinogen mutation A $\alpha$ Arg104 > Cys did not introduce relevant clinical consequences probably due to its low expression levels.

\section{Additional files}

Additional file 1: Control. (MP4 $96 \mathrm{~kb})$

Additional file 2: Father. (MP4 $181 \mathrm{~kb})$

Additional file 3: Proband. (MP4 $113 \mathrm{~kb}$ )

\begin{abstract}
Abbreviations
a.u.: Arbitrary unit; aPTT: Activated partial thromboplastin time; DAB: 3, 3'diaminobenzidine; DTT: Dithiothreitrol; EDTA: Ethylenediaminetetraacetatic acid; EM: Elastic modulus; Fg: Fibrinogen; FXIIla: Activated factor XIII; HAS: Hemostasis analyzer system; Ks: Permeation constant; MaxAbs: Maximum absorbance; OD: Optical density; PCR: Polymerase chain reaction; PPP: Platelet poor plasma; PT: Prothrombin time; SDS/PAGE: Sodium dodecyl sulfate polyacrylamide gel electrophoresis; TBS: Tris buffered saline; TF: Tissue factor; TOF-MS: Reverse phase-electrospray time of flight-mass spectrometry; tPA: Tissue type plasminogen activator; TT: Thrombin time
\end{abstract}

\section{Acknowledgements}

We are grateful to the patients of the present study for their collaboration.

\section{Funding}

The investigation has been partially supported by the government of Bolivarian Republic of Venezuela.

\section{Availability of data and materials}

Most data generated or analyzed during this study are included in this published article (and its supplementary information files). Other datasets used and/or analyzed during the current study and not shown are available from the corresponding author on reasonable request.

\section{Authors' contributions}

MR formulated research goal and aims, performed the analysis of data and wrote the article. LM performed the biochemical and structural fibrinogen variant characterization experiments. $\mathrm{RH}$ performed the confocal microscopy experiments, the images data analysis, and figures editing. S-RA performed the clinical evaluation of the patient. MM sequenced the DNA of the patients. CA performed the experiments of dynamic fibrin clot growth, some analytical determinations, analysis of the data, and participated in the writing of the article. BS participated in the formulation of goal and aims, and mass spectrometry studies, analysis of the data, and writing of the article. All authors read and approved the final manuscript and gave their consent for publication.

\section{Ethics approval and consent to participate}

The Ethics Committee of the Instituto Venezolano de Investigaciones Científicas (IVIC) approved the project under the number DIR-1925/1579/ 2016. All subjects signed an informed consent before blood withdrawal, and written parental consent was obtained for the proband.

\section{Consent for publication}

Not applicable.

\section{Competing interests}

The authors declare that they have no competing interests.

\section{Publisher's Note}

Springer Nature remains neutral with regard to jurisdictional claims in published maps and institutional affiliations.

\section{Author details}

${ }^{1}$ Lab. Biología del Desarrollo de la Hemostasia. Instituto Venezolano de Investigaciones Científicas (IVIC), Caracas, Bolivarian Republic of Venezuela. ${ }^{2}$ Instituto de Inmunología, Universidad Central de Venezuela and Lab. Fisiología Celular Centro de Biofisica y Bioquímica (IVIC), Caracas, Bolivarian Republic of Venezuela. ${ }^{3}$ Banco Municipal de Sangre del Distrito Capital, Caracas, Bolivarian Republic of Venezuela. ${ }^{4}$ Medical Engineering and Biotechnology, University of Applied Sciences, Jena, Germany. ${ }^{5}$ Division of Angiology and Haemostasis, Faculty of Medicine, University Hospitals of 
Geneva, Geneva, Switzerland. ${ }^{6}$ Molecular Pathology Laboratory, University of Otago, Christchurch, New Zealand.

Received: 25 April 2017 Accepted: 29 August 2017

Published online: 22 December 2017

\section{References}

1. Blomback B, Hessel B, Hogg D, Therkildsen L. A two-step fibrinogen-fibrin transition in blood coagulation. Nature. 1978;275:501-5.

2. Asselta R, Duga S, Tenchini ML. The molecular basis of quantitative fibrinogen disorders. J Thromb Haemost. 2006:4:2115-29.

3. Doolittle RF, Goldbaum DM, Doolittle LR. Designation of sequences involved in the "coiled-coil" interdomainal connections in fibrinogen: constructions of an atomic scale model. J Mol Biol. 1978;120:311-25.

4. Neerman-Arbez M, de Moerloose P, Casini A. Laboratory and genetic investigation of mutations accounting for congenital fibrinogen disorders. Semin Thromb Hemost. 2016:42:356-65.

5. Cunningham MT, Brandt JT, Laposata M, Olson JD. Laboratory diagnosis of dysfibrinogenemia. Arch Pathol Lab Med. 2002;126:499-505.

6. Casini A, Brungs T, Lavenu-Bombled C, Vilar R, Neerman-Arbez M, de Moerloose P. Genetics, diagnosis and clinical features of congenital hypodysfibrinogenemia: a systematic literature review and report of a novel mutation. J Thromb Haemost. 2017;15:876-88

7. Maghzal GJ, Brennan SO, Homer VM, George PM. The molecular mechanisms of congenital hypofibrinogenaemia. Cell Mol Life Sci. 2004;61:1427-38.

8. Zhang JZ, Redman CM. Role of interchain disulfide bonds on the assembly and secretion of human fibrinogen. J Biol Chem. 1994;269:652-8.

9. Zhang JZ, Redman C. Fibrinogen assembly and secretion. Role of intrachain disulfide loops. J Biol Chem. 1996;271:30083-8.

10. Xu W, Chung DW, Davie EW. The assembly of human fibrinogen. The role of the amino-terminal and coiled-coil regions of the three chains in the formation of the alphagamma and betagamma heterodimers and alphabetagamma half-molecules. J Biol Chem. 1996;271:27948-53.

11. Ingram IC. The determination of plasma fibrinogen by the clot-weight method. Biochem J. 1952;51:583-5.

12. Niwa K, Takebe M, Sugo T, Kawata Y, Mimuro J, Asakura S, et al. A gamma Gly-268 to Glu substitution is responsible for impaired fibrin assembly in a homozygous dysfibrinogen Kurashiki I. Blood. 1996;87:4686-94.

13. Marchi R, Walton BL, McGary CS, Lin FC, Ma AD, Pawlinski R, et al. Dysregulated coagulation associated with hypofibrinogenaemia and plasma hypercoagulability: implications for identifying coagulopathic mechanisms in humans. Thromb Haemost. 2012;108:516-26.

14. Marchi R, Carvajal Z, Meyer M, Soria J, Ruiz-Saez A, Arocha-Pinango CL, et al. Fibrinogen Guarenas, an abnormal fibrinogen with an Aalpha-chain truncation due to a nonsense mutation at Aalpha 467 Glu (GAA)->stop (TAA). Thromb Res. 2006;118:637-50.

15. Towbin H, Staehelin T, Gordon J. Electrophoretic transfer of proteins from polyacrylamide gels to nitrocellulose sheets: procedure and some applications. Proc Natl Acad Sci U S A. 1979;76:4350-4.

16. Marchi R, Echenagucia M, Meyer M, Acosta M, Apitz R, Ruiz-Sáez A. Fibrinogen Maracaibo: Hypo-Dysfibrinogenemia Caused by a Heterozygous Mutation in the Gen that Encodes for the Fibrinogen Aa Chain (G.1194G>A: P.Gly13>Glu) with Diminished Thrombin Generation. J Blood Disorders Transf. 2014;5:1-5.

17. Carter AM, Cymbalista CM, Spector TD, Grant PJ. Heritability of clot formation, morphology, and lysis: the EuroCLOT study. Arterioscler Thromb Vasc Biol. 2007;27:2783-9.

18. Brennan SO, Mangos H, Faed JM. Benign FGB (148Lys->Asn, and 448Arg$>$ Lys), and novel causative gamma211Tyr->his mutation distinguished by time of flight mass spectrometry in a family with hypofibrinogenaemia. Thromb Haemost. 2014;111:679-84.

19. Pieters M, Undas A, Marchi R, De Maat MP, Weisel J, Ariens RA. An international study on the standardization of fibrin clot permeability measurement: methodological considerations and implications for healthy control values. J Thromb Haemost. 2012;10:2179-81.

20. Blomback B, Okada M. Fibrin gel structure and clotting time. Thromb Res. 1982:25:51-70

21. Potze W, Adelmeijer J, Porte RJ, Lisman T. Preserved clot formation detected by the Thrombodynamics analyzer in patients with cirrhosis. Thromb Res. 2015;135:1012-6

22. Panteleev MA, Ovanesov MV, Kireev DA, Shibeko AM, Sinauridze El, Ananyeva NM, et al. Spatial propagation and localization of blood coagulation are regulated by intrinsic and protein $C$ pathways, respectively. Biophys J. 2006:90:1489-500.

23. Riedelova-Reicheltova Z, Kotlin R, Suttnar J, Geierova V, Riedel T, Majek P, et al. A novel natural mutation AalphaPhe98lle in the fibrinogen coiled-coil affects fibrinogen function. Thromb Haemost. 2014;111:79-87.

24. Kotlin R, Reicheltova Z, Maly M, Suttnar J, Sobotkova A, Salaj P, et al. Two cases of congenital dysfibrinogenemia associated with thrombosis - fibrinogen Praha III and fibrinogen Plzen. Thromb Haemost. 2009;102:479-86.

25. Krobot K, Hense HW, Cremer P, Eberle E, Keil U. Determinants of plasma fibrinogen: relation to body weight, waist-to-hip ratio, smoking, alcohol, age, and sex. Results from the second MONICA Augsburg survey 19891990. Arterioscler Thromb. 1992;12:780-8.

26. Hager K, Felicetti M, Seefried G, Platt D. Fibrinogen and aging. Aging (Milano). 1994;6:133-8.

27. Spada RS, Toscano G, Chiarenza S, Di Mauro S, Cosentino Fl, lero I, et al. Ischemic stroke and fibrinogen in the elderly. Arch Gerontol Geriatr Suppl. 2004;9:403-6.

28. Brennan SO, Davis RL, Lowen R, Ruskova A. Deletion of five residues from the coiled coil of fibrinogen (Bbeta Asn167_Glu171del) associated with bleeding and hypodysfibrinogenemia. Haematologica. 2009;94:585-8.

\section{Submit your next manuscript to BioMed Central and we will help you at every step:}

- We accept pre-submission inquiries

- Our selector tool helps you to find the most relevant journal

- We provide round the clock customer support

- Convenient online submission

- Thorough peer review

- Inclusion in PubMed and all major indexing services

- Maximum visibility for your research

Submit your manuscript at www.biomedcentral.com/submit
) Biomed Central 